\title{
Manipulation of Individual Lithographically Defined Silicon Nanowires for Transmission Electron Microscopy
}

\author{
Herman C. Floresca*, Krutarth Trivedi**, Walter Hu** and M. J. Kim* \\ * Department of Materials Science and Engineering, University of Texas, Dallas, Richardson, TX \\ 75080 \\ ** Department of Electrical Engineering, University of Texas, Dallas, Richardson, TX 75080
}

In this study, we have developed a process that allows us to transfer lithographically defined silicon nanowires using a nanomanipulator onto a transmission electron microscope (TEM) grid for planview and side-view. Combined with a cross-section sample, this process gives us a way to analyze the structure of a nanowire from three orthogonal directions.

The silicon nanowires were fabricated from a silicon on insulator (SOI) wafer using electron beam lithography and an inductively coupled plasma (ICP) etch. To allow for manipulation, the nanowires had to be removed from the oxide surface. This was done using a buffered oxide etch (BOE) which removed all the oxide including the e-beam resist around the silicon nanowire, leaving it suspended and anchored only by the square pads at its ends. Manipulation was done inside of a dual beam scanning electron microscope (SEM)/ focused ion beam (FIB). A nanomanipulator with piezoelectric fine movement was attached to the stage to allow the probing of the nanowires.

A suspended nanowire was chosen for transfer and freed from its anchors by breaking its ends, causing the nanowire to fall to the substrate. The probe was then lowered onto the nanowire where static forces took over attaching the nanowire to the probe, allowing for it to be lifted off of the surface, as seen in Fig. 1(a) and 1(b). The probe was then manipulated over to a TEM grid where the nanowire was brought into contact with one of its copper fingers. To overcome the static force attaching the wire to the probe, the nanowire had to be 'welded' to the copper. The electron beam was focused onto the end of the nanowire where it was touching the grid and left for a few seconds. This gave enough time for carbon growth to hold the wire to the grid. After a few seconds, the nanoprobe was slowly moved away and detached from the nanowire, leaving the nanowire attached to the grid. This was done to two nanowires: one for examining the side-view and one for examining the plan-view. Fig. 1(c) shows a nanowire attached to a TEM grid under an SEM.

In Fig. 2(a), the plan view of a nanowire can be seen with a width of about 35 to $40 \mathrm{~nm}$. The inset shows the [001] selected area diffraction (SAD) of the nanowire, reinforcing the fact that the nanowire was made from a single crystal origin. From the images, it appears that the ICP etch on the nanowire does not leave an atomically smooth surface. Fig. 2 (b) shows the side view of a nanowire fabricated on the same sample with a height of about $60 \mathrm{~nm}$. The inset shows a single crystal [110] SAD since the nanowire was fabricated with its axis along the [1-10] direction. From this view it is seen that the nanowire has an atomically flat top and bottom. It makes sense that this would be the case since the SOI top silicon layer is atomically smooth and that the ICP never reaches either of these surfaces due to the buried oxide layer and e-beam resist protection. For comparison, Fig. 2(c) is a cross-section made of a nanowire on the same sample with a width and height of about 40 and $60 \mathrm{~nm}$ respectively. 
Using this manipulation and preparation process, multiple views of nanowires can be taken, allowing a comprehensive examination of the shape and structure. Without multiple views, an image of only the nanowire's side view may lead one to assume that its top and bottom are exactly the same as its sides when, as seen in this study, they may be completely different.

\section{References}

[1] This work was supported by a grant from the State of Texas ETF (FUSION)
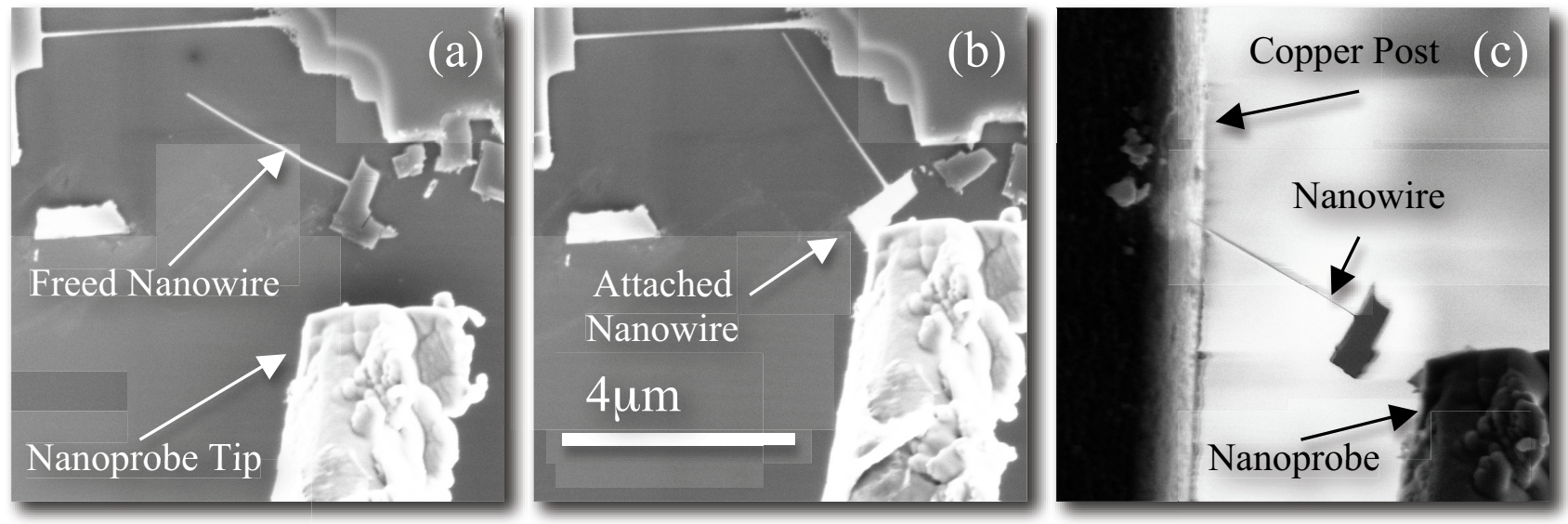

FIG. 1. (a) A nanoprobe is brought close to a freed nanowire. (b) The nanowire has been electrostatically attached to the nanoprobe and lifted off the surface. (c) Attached nanowire to the TEM grid with the nanoprobe detatched.

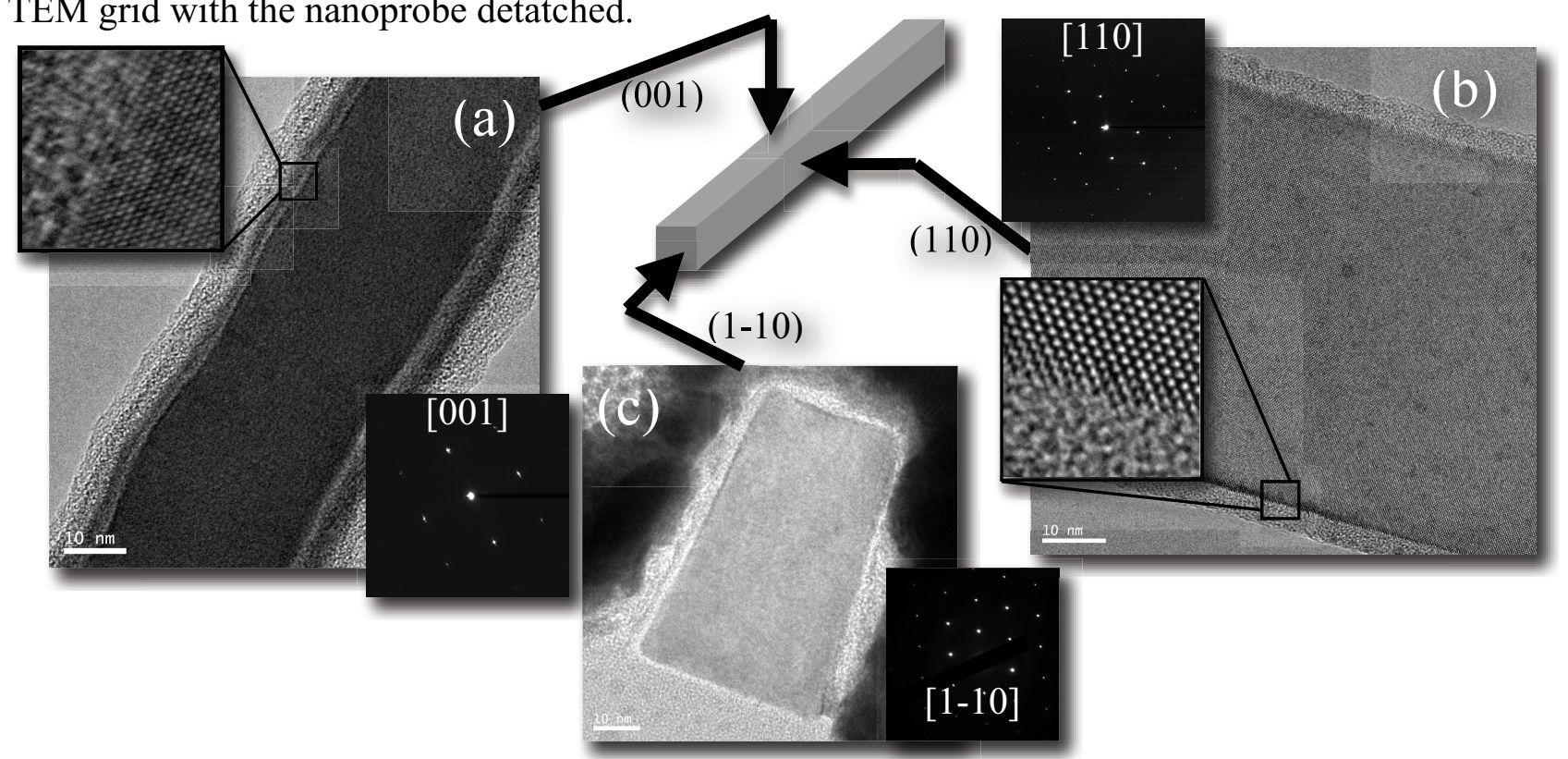

FIG. 2. (a) $<100>$ Plan-view of nanowire. Sides are seen to not have atomically flat surfaces. (b) Side-view of nanowire with top and bottom surfaces atomically flat. (c) Cross-section shape of nanowire showing the $40 \mathrm{~nm}$ width and $60 \mathrm{~nm}$ height reflected in (a) and (b). 